\title{
ON THE COHOMOLOGICAL EQUIVALENCE OF A CLASS OF FUNCTIONS UNDER AN IRRATIONAL ROTATION OF BOUNDED TYPE
}

\author{
LAWRENCE BAGGETT AND KATHY MERRILL
}

(Communicated by J. Marshall Ash)

\begin{abstract}
We prove that a linear combination of positive real powers of $x$, with integral 0 and equal values at 0 and 1 , is a coboundary for any irrational rotation of bounded type. We apply this result to establish the ergodicity of related compact and noncompact skew products.
\end{abstract}

\section{INTRODUCTION}

We shall study an equivalence relation defined on real-valued functions on $\mathbb{R} / \mathbb{Z}$. Two functions, $v_{1}$ and $v_{2}$, are cohomologous if there exists a Lebesgue measurable, real-valued function $w$ such that

$$
v_{1}(x)-v_{2}(x)=w(x)-w(x+\theta)
$$

for almost all $x$, where $\theta$ is a fixed irrational.

This equivalence relation arises in the representation theory of nontype I groups $[3,8]$ and groups with ordered duals [6]. It is also related to the ergodicity of skew products of the form

$$
\begin{gathered}
T_{v}: \mathbb{R} / \mathbb{Z} \times \mathbb{R} \rightarrow \mathbb{R} / \mathbb{Z} \times \mathbb{R} \\
T_{v}(x, y)=(x+\theta, y+v(x)) .
\end{gathered}
$$

If $v$ is cohomologous to a constant $c$, then $f(x, y)=e^{2 \pi i(w(x)+y) / c}$ is an invariant function for $T_{v}$, thus showing that $T_{v}$ is not ergodic. If $v$ is cohomologous to a nonconstant function $w$, then the ergodicity of $T_{w}$ implies the ergodicity of $T_{v}$ [9].

We will focus our attention on the cohomology relations among functions of the form $x^{\alpha}-1 /(\alpha+1)$, for $\alpha>0$, and on the ergodicity of the corresponding skew products $T_{x^{\alpha}-1 /(\alpha+1)}$. (The constant $1 /(\alpha+1)$ is required since cohomological equivalence requires equal integrals and ergodicity requires an

Received by the editors July 14, 1989 and, in revised form, February 19, 1990; presented by the second author at the American Mathematical Society Annual Meeting, January 17, 1990.

1980 Mathematics Subject Classification (1985 Revision). Primary 28D05; Secondary 11A55, $22 \mathrm{D} 30$. 
integral of 0.) Our results extend easily to linear combinations of functions of this type. We also obtain information about the multiplicative cohomology relations among the exponentials of these functions, and about the ergodicity of the compact skew products these exponentials define.

Previous results concerning cohomology of continuous functions required an $L^{2}$ derivative [2], and thus provided information only for $\alpha>1 / 2$. Previous ergodicity results required the even more restrictive condition of a continuous derivative [5], so that $\alpha \geq 1$. In this paper, we establish the cohomological equivalence of the $x^{\alpha}-1 /(\alpha+1)$ for all $\alpha>0$, and we obtain as a corollary the ergodicity of $T_{x^{\alpha}-1 /(\alpha+1)}$ for all $\alpha>0$. However, we need to restrict the fixed irrational $\theta$ to be of bounded type. This means that there exists a $\delta>0$ such that $\left|1-e^{2 \pi i n \theta}\right| \geq \delta / n$ for all $n>0$. Some restriction has been shown to be essential for cohomology results; the only pairs of continuous functions that are cohomologous for all $\theta$ are those that differ by trigonometric polynomials [1]. Whether the restriction is necessary for the ergodicity result below is still unknown.

2.

We will establish cohomological equivalence, as in [2], by using Fourier techniques to find solutions to the functional equation (1). We write

$$
v_{1}(x)-v_{2}(x)=\sum c_{n}\left(v_{1}-v_{2}\right) e^{2 \pi i n x},
$$

and look for a solution $w(x)=\sum c_{n}(w) e^{2 \pi i n x}$. Suppose that $v_{1}$ and $v_{2}$ are absolutely continuous, with $c_{0}\left(v_{1}-v_{2}\right)=0$, and that $v_{1}(0)-v_{2}(0)=v_{1}(1)-$ $v_{2}(1)$. Then from (1), we see that we should look for a $w$ with $c_{n}(w)=$ $c_{n}\left(v_{1}-v_{2}\right) /\left(1-e^{2 \pi i n \theta}\right)$. The obvious difficulty lies in showing the convergence of $\sum c_{n}(w) e^{2 \pi i n x}$ for this $w$. If $v_{1}-v_{2}$ has an $L^{2}$ derivative, we write, for $n \neq 0, c_{n}(w)=c_{n}\left(v_{1}^{\prime}-v_{2}^{\prime}\right) \sigma_{n}$, where $\sigma_{n}=1 / 2 \pi i n\left(1-e^{2 \pi i n \theta}\right)$, and note that $\left\{\sigma_{n}\right\}$ is a bounded sequence for $\theta$ of bounded type. To extend this technique to functions with $L^{p}$ derivatives, $1<p<2$, we use the following classical result.

Theorem (Marcinkiewicz). Let $\left\{m_{n}\right\}$ be a bounded sequence on $\mathbb{Z}$. Then $\left\{m_{n}\right\}$ is a bounded multiplier on $L^{p}, p>1$, if there exists a lacunary sequence $\left\{N_{k}\right\}$ and a constant $M$ such that $\sum_{N_{k} \leq|j|<N_{k+1}}\left|m_{j}-m_{j+1}\right| \leq M$ for all $k$.

Proof. See [4].

We will take $\left\{N_{k}\right\}$ to be the sequence of denominators of the convergents of the continued fraction expansion for $\theta$. The convergents give the best rational approximations to $\theta$ (best relative to size of denominator). The theory of continued fractions gives estimates on how close these approximations are, and ties the property of being of bounded type to a growth condition for the $\left\{N_{k}\right\}$. In particular, we will need the following facts. 
Lemma 1. For $\theta$ of bounded type, there exist positive constants $\delta$ (as in the definition of bounded type), $\delta^{\prime}$ and $\delta^{\prime \prime}$ such that:

(1) For all $j \leq N_{k}$,

$$
\delta / N_{k} \leq \min _{\substack{0 \leq h \leq N_{k} \\ h \neq j}}\left|e^{2 \pi i j \theta}-e^{2 \pi i h \theta}\right| \leq \delta^{\prime} / N_{k} .
$$

(2) $\delta^{\prime \prime} N_{k-1} \geq N_{k} \geq N_{k-1}+N_{k-2}$.

Proof. See [7].

Note that by (2), $N_{k} / N_{k-1} \geq 1+1 / \delta^{\prime \prime}>1$, which shows that the $\left\{N_{k}\right\}$ forms a lacunary sequence.

The following lemma uses these properties of the convergents to give an estimate we will need in applying the Marcinkiewicz Theorem to show cohomological equivalence.

Lemma 2. There exist constants $M_{1}$ and $M_{2}$ such that

$$
M_{1} \ln \left(N_{k}\right) \leq \sum_{N_{k} \leq|j|<N_{k+1}}\left|\sigma_{j}-\sigma_{j+1}\right| \leq M_{2} \ln \left(N_{k}\right) .
$$

Proof. By symmetry, we need only consider positive $j$. Now,

$$
\begin{aligned}
\left|\sigma_{j}-\sigma_{j+1}\right| & =\frac{1}{2 \pi}\left|\frac{1}{j(j+1)\left(1-e^{2 \pi i j \theta}\right)}-\frac{e^{2 \pi i j \theta}\left(e^{2 \pi i \theta}-1\right)}{(j+1)\left(1-e^{2 \pi i j \theta}\right)\left(1-e^{2 \pi i(j+1) \theta}\right)}\right| \\
& \geq\left|\frac{\sigma_{j+1}\left(e^{2 \pi i \theta}-1\right)}{1-e^{2 \pi i j \theta}}\right|-\frac{1}{2 \pi \delta(j+1)} .
\end{aligned}
$$

Thus, it will suffice, by (2) of Lemma 1 , to prove the assertion with $\sigma_{j}-\sigma_{j+1}$ replaced by $\sigma_{j+1}$.

We define the sequence $\left\{j_{n}\right\}$ to be a reordering of the integers $N_{k}, N_{k}+$ $1, \ldots, N_{k+1}-1$, so that $j_{1} \theta<j_{2} \theta \cdots<j_{r} \theta<1 / 2<j_{r+1} \theta<\cdots<j_{\left(N_{k+1}-N_{k}\right)} \theta$ $(\bmod 1)$. Then

$$
\begin{aligned}
\left|1-e^{2 \pi i j_{n} \theta}\right| & \geq \frac{2}{\pi}\left(\left|1-e^{2 \pi i j_{1} \theta}\right|+\left|e^{2 \pi i j_{1} \theta}-e^{2 \pi i j_{2} \theta}\right|+\cdots+\left|e^{2 \pi i j_{n-1} \theta}-e^{2 \pi i j_{n} \theta}\right|\right) \\
& \geq 2 n \delta / \pi N_{k}, \quad \text { for } 1 \leq n \leq r,
\end{aligned}
$$

and

$$
\begin{aligned}
\left|1-e^{2 \pi i j_{n} \theta}\right| \geq \frac{2}{\pi}\left(\left|1-e^{2 \pi i j_{\left(N_{k+1}-N_{k}\right)} \theta}\right|+\left|e^{2 \pi i j_{\left(N_{k+1}-N_{k}\right)} \theta}-e^{2 \pi i j_{\left(N_{k+1}-N_{k}-1\right)} \theta}\right|\right. \\
\left.+\cdots+\left|e^{2 \pi i j_{n+1} \theta}-e^{2 \pi i j_{n} \theta}\right|\right) \\
\geq 2\left(N_{k+1}-N_{k}-n\right) \delta / \pi N_{k}, \quad \text { for } r<n \leq N_{k+1}-N_{k} .
\end{aligned}
$$


Thus,

$$
\begin{aligned}
\sum_{N_{k} \leq|j|<N_{k+1}}\left|\sigma_{j}\right| & =\sum_{n=1}^{N_{k+1}-N_{k}} \frac{1}{2 \pi j_{n}\left|1-e^{2 \pi i j_{n} \theta}\right|} \\
& \leq 2 \sum_{n=1}^{N_{k+1}-N_{k}} \frac{N_{k}}{4 \delta n j_{n}} \\
& \leq 2 \sum_{n=1}^{N_{k+1}-N_{k}} \frac{1}{4 \delta n} \\
& \leq M_{2} \ln \left(N_{k}\right)
\end{aligned}
$$

for some constant $M_{2}$.

Now for the lower bound, we note similarly that $\left|1-e^{2 \pi i j_{n} \theta}\right| \leq n \delta^{\prime} / N_{k}$ for $1 \leq n \leq N_{k+1}-N_{k}$, so that

$$
\begin{aligned}
\sum_{n=1}^{N_{k+1}-N_{k}} \frac{1}{2 \pi j_{n}\left|1-e^{2 \pi i j_{n} \theta}\right|} & \geq \sum_{n=1}^{N_{k+1}-N_{k}} \frac{N_{k}}{2 \pi \delta^{\prime} n j_{n}} \\
& \geq \sum_{n=1}^{N_{k+1}-N_{k}} \frac{1}{2 \pi \delta^{\prime \prime} \delta^{\prime} n} \\
& \geq M_{1} \ln \left(N_{k}\right)
\end{aligned}
$$

for some constant $M_{1}$.

We see by Lemma 2 that $\left\{\sigma_{n}\right\}$ does not satisfy the hypothesis of the Marcinkiewicz Theorem. In the following theorem, we apply Marcinkiewicz using an appropriately modified sequence.

Theorem 1. Suppose $v$ is absolutely continuous, $v(0)=v(1)$, and $\left\{\ln (|n|) c_{n}\left(v^{\prime}\right)\right\}$ is the sequence of Fourier coefficients of an $L^{p}$ function, $p>1$. Then $v$ is cohomologous to the constant $c_{0}(v)$.

Proof. We have that $\left\{2 \pi i n \ln (|n|) c_{n}(v)\right\}$ is the sequence of Fourier coefficients of an $L^{p}$ function. Also, as in Lemma 2, we see that $\left\{\sigma_{n} / \ln (|n|)\right\}$ satisfies the hypotheses of the Marcinkiewicz Theorem. Thus $\left\{c_{n}(v) /\left(1-e^{2 \pi i n \theta}\right)\right\}$ is the sequence of Fourier coefficients of an $L^{p}$ function $w$. Then by substitution we see that $v, c_{0}(v)$, and $w$ satisfy (1).

We use Theorem 1 to show the cohomological equivalence of functions of the form $x^{\alpha}-1 /(\alpha+1)$.

Theorem 2. The functions $x^{\alpha}-1 /(\alpha+1)$ and $x^{\beta}-1 /(\beta+1)$ are cohomologous, for any $\alpha, \beta>0$.

Proof. Let $v_{\alpha}(x)=x^{\alpha}-x$. By Theorem 1 , it will suffice to show that $\{\ln (|n|)$ $\left.\times c_{n}\left(v_{\alpha}^{\prime}\right)\right\}$ is the sequence of Fourier coefficients of an $L^{p}$ function, $p>1$. By previous results [2], we need only consider $0<\alpha \leq 1 / 2$. 
We use a change of variables to write, for $n \neq 0, c_{n}\left(v_{\alpha}^{\prime}\right)=\alpha|n|^{-\alpha} I_{n, \alpha}$, where $I_{n, \alpha}=\int_{0}^{|n|} e^{-2 \pi i x(\operatorname{sgn}(n))} x^{\alpha-1} d x$. Choose an $\varepsilon, 0<\varepsilon<\alpha$, and note that $v_{\alpha-\varepsilon}^{\prime}$ is in $L^{p}$ for some $p>1$. Now we have that

$$
\ln (|n|) c_{n}\left(v_{\alpha}^{\prime}\right)=\frac{\alpha \ln (|n|) I_{n, \alpha}}{(\alpha-\varepsilon)|n|^{\varepsilon} I_{n, \alpha-\varepsilon}} c_{n}\left(v_{\alpha-\varepsilon}^{\prime}\right) .
$$

It will suffice to show that each of the three sequences $\left\{\ln (|n|)|n|^{-\varepsilon}\right\},\left\{I_{n, \alpha}\right\}$, and $\left\{1 / I_{n, \alpha-\varepsilon}\right\}$ satisfies the hypotheses of the Marcinkiewicz Theorem. We will use the fact that if a sequence $\left\{m_{n}\right\}$ is eventually monotone, then for large $k$, the Marcinkiewicz sum telescopes, so that

$$
\sum_{N_{k} \leq|n|<N_{k+1}}\left|m_{n}-m_{n+1}\right|=\left|m_{N_{k}}-m_{N_{k+1}}\right|+\left|m_{\left(-N_{k}\right)}-m_{\left(-N_{k+1}\right)}\right| .
$$

Thus, a bounded, eventually monotone sequence gives a bounded multiplier.

This comment is enough to show that the first of our sequences is a bounded multiplier: for $n$ large in absolute value, $\ln (|n|)|n|^{-\varepsilon}$ decreases monotonically as $|n|$ increases. Now we consider the sequence $\left\{I_{n, \alpha}\right\}$. We write

$$
\begin{aligned}
I_{n, \alpha} & =\int_{0}^{|n|} \frac{\cos 2 \pi x}{x^{1-\alpha}} d x \pm i \int_{0}^{|n|} \frac{\sin 2 \pi x}{x^{1-\alpha}} d x \\
& =a_{n}(\alpha) \pm i b_{n}(\alpha) .
\end{aligned}
$$

Clearly, $\left\{b_{n}(\alpha)\right\}$ is increasing as $|n|$ increases. Integration by parts shows that $\left\{a_{n}(\alpha)\right\}$ is also increasing, and that both $\left\{a_{n}(\alpha)\right\}$ and $\left\{b_{n}(\alpha)\right\}$ are bounded. Thus by the remark above, the second sequence is also a bounded multiplier.

Finally, we consider the sequence $\left\{1 / I_{n, \alpha-\varepsilon}\right\}=\left\{\overline{I_{n, \alpha-\varepsilon}} /\left|I_{n, \alpha-\varepsilon}\right|^{2}\right\}$. Now, $\left\{\overline{I_{n, \alpha-\varepsilon}}\right\}$ is a bounded multiplier by the same argument as above. Also, $\left|I_{n, \alpha-\varepsilon}\right|^{2}$ $=\left(a_{n}(\alpha-\varepsilon)\right)^{2}+\left(b_{n}(\alpha-\varepsilon)\right)^{2}$, so that $\left\{1 /\left|I_{n, \alpha-\varepsilon}\right|^{2}\right\}$ is eventually decreasing, and is thus a bounded multiplier.

Corollary 1. If $v(x)=\sum_{j=1}^{n} \beta_{j} x^{\alpha_{j}}$, where $\beta_{j} \in \mathbb{R}, \alpha_{j} \geq 0$, and if $v(0)=v(1)$, then $v$ is cohomologous to its integral $c_{0}(v)$.

Proof. By Theorem 2, we have that for each $j$, with $\alpha_{j} \neq 0, \beta_{j} x^{\alpha_{j}}-\beta_{j} /\left(\alpha_{j}+1\right)$ is cohomologous to $\beta_{j}(x-1 / 2)$. Thus $v-c_{0}(v)$ is cohomologous to $\left(\sum_{\alpha_{j} \neq 0} \beta_{j}\right)$ $\times(x-1 / 2)$, which is 0 since $v(0)=v(1)$.

Corollary 2. Let $v(x)=\sum_{j=1}^{n} \beta_{j} x^{\alpha_{j}}$, where $\beta_{j} \in \mathbb{R}, \alpha_{j} \geq 0$. If $v(0) \neq v(1)$ and if $v$ has integral 0 , then the skew product $T_{v}$ is ergodic.

Proof. As in Corollary 1 , we see that $v$ is cohomologous to $c(x-1 / 2)$, where $c$ is nonzero. The corollary then follows from the ergodicity of $T_{x-1 / 2}$, which is established in [5].

We now apply our cohomology results to compact skew products. We will 
need the following definitions:

Two circle-valued functions $f_{1}$ and $f_{2}$ defined on $\mathbb{R} / \mathbb{Z}$ are multiplicatively cohomologous if there exists a Lebesgue measurable circle-valued function $g$ such that

$$
f_{1}(x) / f_{2}(x)=g(x) / g(x+\theta)
$$

for almost all $x$. A simple substitution shows that $e^{2 \pi i v_{1}}$ is multiplicatively cohomologous to $e^{2 \pi i v_{2}}$ if $v_{1}$ is cohomologous to $v_{2}$.

A circle-valued function $f$ on $\mathbb{R} / \mathbb{Z}$ defines a compact skew product

$$
\begin{gathered}
S_{f}: \mathbb{R} / \mathbb{Z} \times \mathbb{T} \rightarrow \mathbb{R} / \mathbb{Z} \times \mathbb{T}, \\
S_{f}(x, \beta)=(x+\theta, \beta f(x)) .
\end{gathered}
$$

Just as with noncompact skew products, $f_{1}$ cohomologous to $f_{2}$ implies that the corresponding skew products $S_{f_{1}}$ and $S_{f_{2}}$ share either ergodicity or lack of ergodicity. The compact case is simpler than the noncompact in that a Fourier series argument can be used to show that $S_{f}$ is ergodic if and only if $f^{k}$ is not cohomologous to 1 for any nonzero integer $k$.

Applying Corollary 1 now gives us the following corollary.

Corollary 3. If $v(x)=\sum_{j=1}^{n} \beta_{j} x^{\alpha_{j}}$, where $\beta_{j} \in \mathbb{R}, \alpha_{j} \geq 0$, then the compact skew product $S_{e^{2 \pi i v}}$ is ergodic if and only if either

(1) $v(0) \neq v(1)$

or

(2) $v(0)=v(1)$ and $\int v \neq p+q \theta$ for any $p, q \in \mathbb{Q}$.

Proof. If $v(0) \neq v(1)$, define a nonconstant linear function $u$ by $u(x)=$ $x v(1)+(1-x) v(0)$. Then by Corollary $1, v-u$ is cohomologous to a constant $c$, so that $e^{2 \pi i v}$ is multiplicatively cohomologous to $e^{2 \pi i(u+c)}$. By [3], $e^{2 \pi i k(u+c)}$ cannot be cohomologous to 1 for nonconstant $u$ and $k \neq 0$.

If $v(0)=v(1)$ then by Corollary $1, v$ is cohomologous to its integral $c_{0}(v)$. Thus $e^{2 \pi i v}$ is multiplicatively cohomologous to $e^{2 \pi i c_{0}(v)}$, so that $S_{e^{2 \pi i v}}$ is ergodic if and only if $S_{e^{2 \pi i c_{0}(v)}}$ is ergodic. The latter is ergodic if and only if $e^{2 \pi i k c_{0}(v)}$ is not cohomologous to 1 for any integer $k \neq 0$. This is true if and only if $e^{2 \pi i k c_{0}(v)}$ is not an eigenvalue for rotation by $\theta$, so not of the form $e^{2 \pi i j \theta}$ for any integers $j$ and $k \neq 0$.

\section{ACKNOWLEDGMENT}

The authors wish to thank Professor W. Schmidt for helpful conversations.

\section{REFERENCES}

1. L. Baggett, On functions that are trivial cocycles for a set of irrationals, Proc. Amer. Math. Soc. 104 (1988), 1211-1215.

2. L. Baggett and K. Merrill, Equivalence of cocycles under an irrational rotation, Proc. Amer. Math. Soc. 104 (1988), 1050-1053. 
3. Representations of the Mautner group and cocycles of an irrational rotation, Michigan Math. J. 33 (1986), 221-229.

4. R. E. Edwards and G. I. Gaudry, Littlewood-Paley and multiplier theory, Springer-Verlag, Berlin and New York, 1977.

5. P. Hellekalek and G. Larcher, On the ergodicity of a class of skew products, Israel J. Math. 54 (1986), 301-306.

6. H. Helson, Analyticity on compact abelian groups, Algebras in Analysis, Academic Press, New York, 1975, pp. 1-62.

7. A. Ya. Khinchin, Continued fractions, University of Chicago Press, Chicago, 1964.

8. A. Ramsay, Nontransitive quasiorbits in Mackey's analysis of group extensions, Acta Math. 137 (1976), 17-48.

9. K. Schmidt, Cocycles of ergodic transformation groups, Lecture Notes in Math., vol. 1, MacMillan, India, 1977.

Department of Mathematics, University of Colorado, Boulder, Colorado 80309

Department of Mathematics, The Colorado College, Colorado Springs, Colorado 80903 